\title{
ATITUDES EM RELAÇÃO A PRODUTOS BRASILEIROS: UMA INVESTIGAÇÃO COM EST'UDANTES HOLANDESES
}

\section{ATTITUDE TOWARDS BRAZILIAN PRODUCTS: AN INVESTIGATION WITH DUTCH STUDENTS}

\author{
JANAINA DE MOURA ENGRACIA GIRALDI \\ Doutora em Administração pela Faculdade de Economia, Administração \\ e Contabilidade da Universidade de São Paulo (FEA/USP). \\ Professora Doutora da Faculdade de Economia, Administração \\ e Contabilidade de Ribeirão Preto, Universidade de São Paulo (FEA-RP).
}

Av. dos Bandeirantes, 3900, bloco A, sala 58, Monte Alegre - Ribeirão Preto - SP - CEP 14040-900

E-mail: jgiraldi@usp.br

ANA AKEMI IKEDA

Doutora em Administração pela Faculdade de Economia, Administração

e Contabilidade da Universidade de São Paulo (FEA/USP).

Professora Livre-Docente da Faculdade de Economia, Administração

e Contabilidade da Universidade de São Paulo (FEA/USP).

Av. Prof. Luciano Gualberto, 908, sala E 104, Cidade Universitária - São Paulo - SP - CEP 05508-900

E-mail: anaikeda@usp.br

DIRCEU TORNAVOI DE CARVALHO

Doutor em Administração pela Faculdade de Economia, Administração

e Contabilidade da Universidade de São Paulo (FEA/USP).

Professor Livre-Docente da Faculdade de Economia, Administração

e Contabilidade de Ribeirão Preto, Universidade de São Paulo (FEA-RP).

Av. dos Bandeirantes, 3900, Monte Alegre - Ribeirão Preto - SP - CEP 14040-900

E-mail: tornavoi@usp.br 


\section{RESUMO}

Pelo fato de a atividade de comércio internacional estar se tornando uma parte central da economia mundial, existe uma necessidade cada vez maior de estudar as atitudes dos consumidores com relação a produtos domésticos e estrangeiros. Nesse sentido, o presente artigo explora como um segmento de consumidores estrangeiros avalia alguns produtos brasileiros: carne bovina, frutas frescas, calçados e móveis. Os objetivos são verificar as atitudes com relação aos produtos brasileiros citados e as possíveis diferenças nas atitudes. Para tanto, é empreendida uma pesquisa descritiva, coletando dados pelo método transversal simples de uma amostra da população de estudantes universitários holandeses. Em conclusão, verificou-se que os produtos brasileiros analisados possuem diferentes avaliações dos respondentes, com exceção das atitudes relacionadas aos móveis e à carne bovina. Os produtos que obtiveram as melhores avaliações foram as frutas frescas, e os que receberam as piores avaliações foram os calçados, porém o estudo também mostrou que os calçados recebem avaliações mais positivas daqueles respondentes que disseram conhecer o produto.

\section{PALAVRAS=CHAVE}

Atitudes; Comportamento do consumidor; Produtos brasileiros; Exportação; Marketing internacional.

\section{ABSTRACT}

Since the international trade activity is becoming a central part of the world economy, there is an increasing need to study the attitudes consumers have towards domestic and foreign products. In this regard, the present paper explores how a segment of foreign consumers evaluates some Brazilian products: cow meet, fresh fruits, shoes and furniture. The objectives are to verify the attitudes towards the cited Brazilian products and to verify the possible differences among these atti- 
tudes. In order to achieve these objectives, it was performed a descriptive research, collecting data by the survey method in a sample of a undergraduate Dutch students population. In conclusion, it was seen that the Brazilian products under analysis have different evaluations from the respondents, with the exception of the attitudes related to furniture and to cow meet. The products that had the best evaluations were fresh fruits and the ones that received the worst evaluations were the shoes. However, the study also pointed out that the shoes received more positive evaluations from the respondents that knew the product.

\section{KEYWORDS}

Attitudes; Consumer behavior; Brazilian products; Exportation; International marketing.

\section{INTRODUÇÃO}

A crescente sofisticação e educação do consumidor global tem tornado a competição internacional entre os produtos cada vez mais intensa, tanto no mercado doméstico como no internacional. A atividade de comércio internacional está se tornando uma parte central da economia mundial, acarretando em maior necessidade de mensurar as atitudes dos consumidores com relação a produtos domésticos e estrangeiros (NETEMEYER; DURVASULA; LICHTENSTEIN, I99I). Dessa forma, muitos governos, associações de comércio, empresas e organizações públicas têm demonstrado interesse nas atitudes dos consumidores com relação a produtos estrangeiros e aos problemas advindos da competição internacional.

As atitudes dos consumidores com relação a produtos estrangeiros constituem importantes considerações para o delineamento de decisões estratégicas para os negócios, especialmente nas áreas de localização internacional de fábricas e de marketing (CORDELL, I993). Tais atitudes podem ser um indicativo dos problemas de marketing enfrentados pelos produtos, em duas importantes formas. Em primeiro lugar, a preferência por produtos importados é considerada uma inimiga dos produtos fabricados domesticamente e da economia local. Em segundo lugar, a discriminação contra produtos importados em determinados países pode criar barreiras invisíveis para a globalização de uma empresa (AGBONIFOH; ELIMIMIAN, I999).

O presente artigo explora como um segmento de consumidores estrangeiros avalia os seguintes produtos brasileiros: carne bovina, frutas frescas, calçados 
e móveis. Os objetivos são: verificar qual a valência das atitudes (positivas ou negativas) com relação aos produtos brasileiros citados e examinar as possíveis diferenças nas atitudes (qual produto possui atitude mais favorável).

Para alcançar os objetivos propostos, foi realizada uma pesquisa empírica com um segmento de consumidores estrangeiros, de modo a coletar as suas avaliações e impressões sobre esses produtos brasileiros. Dessa forma, este trabalho apresenta duas partes. A primeira trata da revisão da literatura sobre o conceito de atitude e sobre as formas de mensuração deste construto. A segunda parte apresenta os resultados de uma investigação empírica da atitude de um segmento de consumidores estrangeiros, a saber, estudantes universitários holandeses, com relação aos produtos brasileiros. Nessa parte, foi empregada a pesquisa descritiva, a qual pode ser conduzida para determinar as crenças, atitudes e opiniões acerca de características dos produtos, mostrando-se adequada para o propósito deste estudo.

Na presente pesquisa, utiliza-se o método transversal simples de pesquisa descritiva (também conhecido como levantamento ou survey), que envolve a coleta de informações de uma determinada amostra apenas uma vez (MALHOTRA, I996). No final do artigo são feitas sugestões para as empresas brasileiras exportadoras, a partir dos resultados obtidos na pesquisa.

\section{REFERENCIAL TEÓRICO: ATITUDES DO CONSUMIDOR}

Em um contexto de comportamento do consumidor, atitude é uma predisposição aprendida para se comportar de forma consistentemente favorável ou desfavorável com relação a um determinado objeto (ASSAEL, I995; SCHIFFMAN; KANUK, I997). O objeto em questão pode ser um produto, categoria de produto, marca, serviço, posse, uso de um produto, pessoas, assuntos, propagandas, preço, mídia ou varejista.

O fato de a atitude ser uma predisposição aprendida significa que ela é formada como resultado de uma experiência direta com o produto, de informações adquiridas de outras pessoas ou da exposição a anúncios. Além disso, as atitudes possuem uma qualidade motivacional, ou seja, elas impelem o consumidor para um determinado comportamento ou o afastam de outro. Para Karsaklian (2004, p. 73), as atitudes têm quatro funções básicas:

- Função instrumental ajustativa: a atitude é formada em torno da recompensa que o indivíduo poderá obter e da forma pela qual poderá reduzir a insatisfação; 
- Função ego-defensiva: a atitude protege o indivíduo, distorcendo a realidade se esta se apresentar incongruente com sua auto-imagem e com seus valores;

- Função de expressão de valores: o indivíduo expressa atitudes apropriadas a seus valores pessoais e seu autoconceito;

- Função de conhecimento: permite construir o universo de forma organizada, reorganizando ou modificando elementos que são percebidos como inconsistentes ou incompletos.

Outra característica das atitudes é o fato de elas serem relativamente consistentes com o comportamento que refletem. Porém, apesar dessa consistência, as atitudes não são necessariamente permanentes; elas mudam com o tempo (SCHIFFMAN; KANUK, I997). Dessa forma, é importante considerar as possíveis influências situacionais nas atitudes e no comportamento do consumidor. Por influências situacionais, compreendem-se os eventos ou as circunstâncias que, em um momento específico, influenciam o relacionamento entre a atitude e o comportamento. Uma situação específica pode levar os consumidores a se comportarem de forma aparentemente inconsistente com as suas atitudes.

Adicionalmente, deve-se destacar o fato de, freqüentemente, as atitudes de um indivíduo levarem-no a raciocinar de uma forma específica. Assim, muitas vezes, o conhecimento dessas atitudes por parte do pesquisador permite que haja uma previsão do comportamento do indivíduo, tanto no que se refere ao consumo quanto no que se refere a outros aspectos da vida (BLUM; NAYLOR, I976).

Rodrigues (I977) também destaca a relação entre atitudes e comportamento, ao definir atitude como uma organização duradoura de crenças e cognições em geral, dotada de carga afetiva pró ou contra um objeto social definido, que predispõe a uma ação coerente com as cognições e os afetos relativos a esse objeto.

Em razão dessa natureza dinâmica das atitudes, deve-se considerar o fato de que atitudes favoráveis em relação a um produto, se negligenciadas, podem gradualmente se deteriorar em um estado menos favorável. Ambas as atitudes positivas e negativas podem se tornar mais neutras simplesmente com o passar do tempo (BLACKWELL; MINIARD; ENGEL, 2005). Um objetivo gerencial freqüente refere-se à mudança das atitudes do consumidor com relação ao produto ou à marca.

Para entender o papel desempenhado pelas atitudes no comportamento do consumidor, deve-se compreender como elas se desenvolvem. As atitudes são desenvolvidas com o tempo, por meio de um processo de aprendizagem afetado pelas influências da família e do grupo de referência do consumidor, das informações recebidas acerca do produto, da experiência passada e de sua personalidade. Isso significa que os consumidores não precisam, necessariamente, con- 
sumir um produto para desenvolver uma atitude com relação a ele. O processo de aprendizagem é afetado, além das experiências vivenciadas pelo consumidor, pelas influências de sua família e do grupo de referência, pelas informações recebidas e pela sua personalidade (ASSAEL, I995).

\subsection{MODELO DE ATITUDE DE TRÊS COMPONENTES}

Para compreender a relação entre atitudes e comportamento, foram elaborados modelos que capturam as dimensões subjacentes de uma atitude, de forma a melhor explicar ou prever o comportamento do consumidor (SCHIFFMAN; KANUK, I997). Um importante modelo de atitude é o chamado modelo de atitude de três componentes. Segundo Day (1972), há uma aceitação geral de que o conceito de atitude está estruturado em três partes componentes: cognitiva, afetiva e conativa ou comportamental. De acordo com esse modelo, as atitudes consistem em três grandes componentes: cognitivo, afetivo e conativo.

O componente cognitivo consiste nas cognições de um indivíduo, ou seja, o conhecimento e as percepções que foram adquiridos pela combinação da experiência direta com o objeto de atitude com informações de várias fontes. Para Schiffman e Kanuk (I997), esse conhecimento e as percepções resultantes podem tomar a forma de crenças, isto é, o consumidor acredita que o objeto de atitude possui vários atributos.

O componente afetivo representa as emoções ou sentimentos dos consumidores com relação a um produto ou marca em particular. Tais emoções e sentimentos são freqüentemente tratados pelos pesquisadores em comportamento do consumidor como avaliativos por natureza. Isso significa que eles capturam uma avaliação direta ou global de um indivíduo com relação ao objeto de atitude.

Finalmente, o componente conativo ou comportamental relaciona-se com a probabilidade com que um indivíduo irá adotar um comportamento específico com relação ao objeto de atitude. Na pesquisa de comportamento do consumidor, o componente conativo é freqüentemente tratado como uma expressão da intenção de compra do consumidor.

Para Assael (I995), a ligação entre esses três componentes ilustra a hierarquia de efeitos de alto envolvimento: as crenças sobre as marcas/produtos influenciam as suas avaliações (afeto), que, por sua vez, influenciam a intenção de compra. Essa hierarquia de efeitos sugere que os profissionais de marketing deveriam influenciar as crenças dos consumidores para encorajar atitudes positivas com relação à marca e para aumentar a chance de o consumidor comprar o produto. 
Por sua vez, os profissionais de marketing podem também trabalhar com as avaliações da marca (componente afetivo da atitude) sem necessariamente influenciar as crenças. Uma tentativa de influenciar as avaliações da marca ou do produto requer o uso de símbolos e imagens para evocar sentimentos e emoções positivos com relação a eles. Influenciar as crenças tende a ser mais baseado em informações, pois essa estratégia tem foco nas características do produto.

Finalmente, o terceiro componente da atitude, a intenção de compra, pode ser abordado sem que as crenças e a avaliação da marca ou do produto sejam influenciadas. Por exemplo, uma grande redução no preço de um produto pode ser um estímulo suficiente para que os consumidores experimentem uma marca menos desejada. As crenças e as atitudes acerca de uma marca escolhida não precisam mudar para que os consumidores estabeleçam uma intenção de compra (ASSAEL, I995).

Conforme observado, existem fatores que levariam à formação e à mudança de atitude, uma vez que elas são aprendidas, sendo afetadas por informações e experiências. Assim, para Wilkie (I994), como há formação e possível mudança de atitude, os profissionais de marketing estão cada vez mais interessados nos estudos referentes a esses temas.

\subsection{RELAÇÃO ENTRE CRENÇAS E ATITUDES}

Uma das principais preocupações dos profissionais de marketing está relacionada com a forma como as crenças de um consumidor influenciam as atitudes com relação à marca. Dentre as teorias que enfocam esse relacionamento, destaca-se a teoria de multiatributo de Fishbein (ASSAEL, I995).

O modelo multiatributo de atitude desenvolvido por Fishbein descreve a formação da atitude como uma função das crenças dos consumidores acerca dos atributos e benefícios de uma marca. Esse modelo de permite aos pesquisadores o diagnóstico das forças e fraquezas de certas marcas com relação à concorrência, ao determinar a forma como os consumidores avaliam as alternativas nos atributos considerados importantes.

De acordo com esse modelo, uma atitude (A) com relação a um objeto (O) depende das crenças (c) de um consumidor acerca dos atributos (i) que esse objeto possui, e das avaliações ou pesos (p) desses atributos (i). Dessa forma,

$$
\mathrm{A}(\mathrm{O})=\sum_{\mathrm{i}=\mathrm{I}}^{\mathrm{n}} \mathrm{ci} \cdot \mathrm{pi}
$$


Pode-se dizer, ao analisar o modelo anteriormente apresentado, que a imagem de um país pode influenciar a atitude com relação aos produtos advindos desse país. Para Fishbein e Ajzen (I975), as crenças sobre os atributos de um produto precedem e influenciam a atitude para com o produto. De acordo com esse modelo, a imagem do país afeta diretamente as crenças acerca dos atributos dos produtos, os quais influenciam a atitude geral para com o produto.

Portanto, o país de origem dos produtos afeta de forma indireta as atitudes por meio das crenças, um fenômeno geralmente descrito como o efeito halo (AYROSA, 2000). Para Nebenzhal, Jaffe e Shlomo (1997), o efeito halo desenvolve-se quando os consumidores sabem pouco sobre os produtos de um país, e suas percepções sobre os atributos dos produtos feitos nesse país são baseadas em qualquer conhecimento que possam ter sobre o país, incluindo o seu nível de desenvolvimento político, econômico e social.

Os estudos sobre os efeitos do país de origem têm procurado identificar processos que possam ajudar a explicar como o país de origem influencia a avaliação de um produto. O construto "país de origem" desenvolve-se a partir da idéia de que as pessoas possuem avaliações estereotipadas sobre países estrangeiros e, conseqüentemente, sobre produtos fabricados nesses países (BALABANIS; MUELLER; MELEWAR, I999). Dessa forma, os estereótipos que os consumidores possuem sobre alguns países podem transcender as avaliações de marcas ou produtos específicos e determinar, até certo ponto, as intenções de compra e o comportamento das pessoas.

Deve-se acrescentar que os efeitos do país de origem nas avaliações de produtos pelo consumidor podem ser mediados por uma variedade de informações intrínsecas (que envolvem a composição física do produto) e extrínsecas (relacionadas ao produto, mas que não fazem parte de sua composição física) sobre o produto, e por fatores ambientais e culturais. Comum à maioria dos estudos sobre indicações múltiplas a respeito do produto é a noção de que as percepções do consumidor são importantes porque irão, no final, influenciar suas escolhas por um produto ou uma marca (CHAO; GUPTA, I995).

Os pesquisadores de marketing e os gerentes de empresas estão interessados em compreender como os consumidores utilizam as informações sobre o país de origem na avaliação de produtos estrangeiros. Os fatores operantes no nível psicológico de um consumidor individual podem fornecer uma compreensão adicional sobre o processamento das informações sobre o país de origem pelos consumidores. Assim, as diferenças individuais na tendência de avaliação podem influenciar o efeito do país de origem. Esse conhecimento é importante, pois pode auxiliar as empresas a adotarem estratégias mais efetivas na comercialização de seus produtos no exterior. 


\subsection{MENSURAÇÃO DE ATITUDES}

Os três componentes da atitude (a cognição, o afeto e a intenção de comportamento) devem ser mensurados nos estudos que envolvem esse construto. Como esses componentes são variáveis qualitativas, essa tarefa é dificultada. A mensuração das atitudes é muito mais difícil e utiliza escalas menos precisas, pois uma atitude é um construto que existe na mente dos consumidores, não sendo observada diretamente, como no caso do peso ou da altura de uma pessoa (MCDANIEL; GATES, I996).

Segundo McDaniel e Gates (1996), o escalonamento de atitudes é baseado em várias definições operacionais criadas para mensurar o construto atitude. Muitas vezes, a atitude é mensurada de forma nominal ou ordinal, mas existem escalas mais sofisticadas que permitem ao pesquisador medir a atitude de forma intervalar.

Para Assael (1995), a abordagem mais comum na mensuração de atitudes é desenvolver escalas de avaliação para que os consumidores possam identificar o grau em que acreditam que um produto possua determinados atributos (as crenças), o grau em que preferem certos produtos (afeto), e as suas intenções de compra.

A presente pesquisa irá utilizar as escalas indicadas por Assael (I995) e McDaniel e Gates (I996) para mensurar os três componentes da atitude do público-alvo com relação aos produtos brasileiros. São também adaptados alguns itens do questionário utilizado por Ayrosa (2002) em sua pesquisa sobre país de origem, visto que algumas questões referem-se às crenças do respondente com relação aos produtos de um país. O Quadro I apresenta as questões correspondentes a cada dimensão de atitude.

A escala apresentada é repetida quatro vezes, uma para cada produto brasileiro pesquisado neste estudo. Os produtos brasileiros objetos dessa pesquisa foram determinados a partir da lista dos principais produtos brasileiros exportados para a Holanda no período entre janeiro e abril de 2004 .

Dentre os produtos de maior volume de exportação, foram escolhidos aqueles que são potencialmente consumíveis pela população-alvo da pesquisa, os estudantes holandeses de graduação da School of Economics Haarlem. A seguir, são detalhados os procedimentos metodológicos da pesquisa empírica. 
QUADRO I

DIMENSÖES DE ATITUDE COM RELAÇÃO A PRODUTOS

UTILIZADOS NA PRESENTE PESQUISA

\begin{tabular}{ll}
\hline COMPONENTES DA ATITUDE & \multicolumn{1}{c}{ QUESTÕES } \\
\hline \multirow{3}{*}{ Cognitivo } & $\begin{array}{l}\text { O produto brasileiro possui boa reputação } \\
\text { O produto brasileiro é caro } \\
\text { O produto brasileiro tem prestígio } \\
\text { O produto brasileiro é de alta qualidade }\end{array}$ \\
& Eu gosto do produto brasileiro \\
Efetivo & Eu acho o produto brasileiro melhor do que o de outros países \\
& Eu admiro o produto brasileiro \\
& Eu tenho simpatia pelos produtos brasileiros \\
\hline Conativo & Eu compraria um produto brasileiro \\
& Eu recomendaria um produto brasileiro a um amigo \\
& Eu prefiro produtos brasileiros a produtos de outros países \\
\hline
\end{tabular}

Fonte: Adaptado de Assael (I995), McDaniel e Gates (I996) e Ayrosa (2002).

\section{PROCEDIMENTOS METODOLÓGICOS/ ESTUDO}

A população desta pesquisa foi definida como sendo os estudantes holandeses da School of Economics Haarlem, Holanda. Essa faculdade oferece os seguintes cursos de graduação: Contabilidade (220 alunos), Marketing (532 alunos), Estudos de Negócios Internacionais (2II alunos), Economia e Logística ( 163 alunos), Gerência, Economia e Direito ( 356 alunos), Gerência de Pequenas e Médias Empresas (4I9 alunos), totalizando I.9or estudantes. O período considerado para a coleta de dados foi o segundo semestre de 2004 .

A população-alvo foi assim definida, pois o acesso aos respondentes era facilitado, uma vez que a pesquisa contou com o apoio de professores da School of Economics Haarlem, Holanda, para a aplicação do questionário elaborado para esta pesquisa. Além disso, os jovens universitários são um segmento de interesse para as empresas, uma vez que se caracterizam como potenciais compradores dos produtos exportados para a Holanda.

Com relação ao país foco do presente estudo, a Holanda foi escolhida, pois é uma importante porta de entrada dos produtos brasileiros na Europa, em razão 
de o porto de Roterdã ser utilizado como entreposto. O país importou do Brasil, em todo o ano de 2004 , US $\$ 5,89$ bilhões FOB (free on board) e de janeiro a outubro de 2005 o total importado foi de US\$ 4,44 bilhões FOB (ALICEWEB, 2005). Com relação à posição da Holanda no ranking dos países compradores dos produtos brasileiros, o país ocupou a terceira posição em 2004 e a quarta em 2005 (RECEITA FEDERAL, 2004).

Definida a população pesquisada, volta-se a atenção para o tipo e o tamanho de amostra para a pesquisa empírica. Como não se conhece o valor da proporção dos estudantes holandeses da School of Economics Haarlem que possuem uma atitude positiva com relação aos produtos brasileiros (uma das principais variáveis da pesquisa), é considerado, para determinação do tamanho da amostra, o valor de $\mathrm{p}=\mathrm{q}=50 \%$. Dessa forma, é elevado ao máximo o número de elementos que constituirão a amostra (MCDANIEL; GATES, I996; TAGLIACARNE, I989). O cálculo resultante é, considerando-se uma margem de erro de $5 \%$ e um intervalo de confiança de $95 \%$ :

$$
\mathrm{n}=\frac{\mathrm{Z}^{2}[\mathrm{p} . \mathrm{q}]}{\mathrm{E}^{2}}=\frac{2^{2}[0,50(1-0,50)]}{0,05^{2}}=400, \text { onde: }
$$

$\mathrm{n}=$ tamanho da amostra

$\mathrm{Z}=$ nível de confiança expressado pelos erros-padrão

$\mathrm{p}=$ proporção dos estudantes holandeses da School of Economics Haarlem que possuem uma atitude positiva com relação aos produtos brasileiros

$\mathrm{q}=\mathrm{I}-\mathrm{p}$

$\mathrm{E}=$ quantidade aceitável de erro de amostragem

Dessa forma, a amostra da presente pesquisa deveria ter 400 alunos. Porém, de acordo com a regra de ajuste para população finita, se o tamanho da amostra é maior do que $5 \%$ do tamanho da população, caso da presente pesquisa, torna-se necessário fazer um ajuste no tamanho da amostra (MCDANIEL; GATES, I996; MATTAR, I999). O cálculo desse ajuste é feito usando a fórmula a seguir:

$\mathrm{n}^{\prime}=\frac{\mathrm{n} \cdot \mathrm{N}}{\mathrm{N}+\mathrm{n}-1}=\frac{400 \cdot 1901}{1901+400-1}=331$, onde:

n' = tamanho da amostra revisado

$\mathrm{n}=$ tamanho da amostra original

$\mathrm{N}=$ tamanho da população 
A amostra, portanto, deveria ter, no mínimo, 33I alunos da School of Economics Haarlem. Definida a população e o tamanho da amostra necessária, deve-se ressaltar o fato de existir o acesso à lista de elementos amostrais (lista de e-mails dos alunos), considerada o arcabouço amostral da pesquisa. Dessa forma, foi possível selecionar aleatoriamente os elementos da amostra, pela base de endereços eletrônicos existentes nas universidades.

As perguntas sobre a atitude com relação aos produtos brasileiros foram respondidas em escalas de somatório de Likert de cinco pontos, nas quais os respondentes selecionam uma resposta a partir de um número limitado de categorias ordenadas (MCDANIEL; GATES, I996). Segundo Malhotra (1996), essa é uma escala de pontuação amplamente utilizada a qual requer que os respondentes indiquem o grau de concordância ou discordância em relação a uma série de afirmações.

A construção de uma escala de somatório de Likert envolve a elaboração inicial de um extenso conjunto de afirmações que reflitam qualidades ou características do objeto de estudo que possivelmente influenciem a atitude da pessoa em relação a ele (CHURCHILL, I99I). O refinamento da escala é feito de forma a manter apenas as afirmações com maior capacidade de discriminar entre atitudes favoráveis e desfavoráveis. A presente pesquisa, contudo, utilizou apenas escalas já desenvolvidas e validadas em outros países, reportadas na literatura.

A escala de somatório de Likert é muito popular pela facilidade de construção e aplicação (AAKER; DAY, I983). Uma grande desvantagem da escala, apontada por vários autores, é o fato de ela ser essencialmente ordinal (MATTAR, I999; AAKER; DAY, I983). Entretanto, existe controvérsia se a mensuração de atitudes pode ser feita com escalas ordinais ou intervalares. Para Churchill (I99I), as escalas de atitudes certamente não são do tipo razão, já que sua origem não é natural. Na pesquisa aqui relatada, os dados foram coletados por meio da escala de somatório de Likert, considerada ordinal. Portanto, serão adotados procedimentos não-paramétricos de análise dos dados.

Um teste estatístico não-paramétrico é um teste cujo modelo não especifica condições sobre os parâmetros da população da qual se extraiu a amostra. Por isso, os testes não-paramétricos também são chamados de testes livres de distribuição (SIEGEL, I975). A maior parte dos testes não-paramétricos se aplica a dados em escala nominal ou ordinal, sendo, assim, adequados para o tratamento dos dados desta pesquisa.

Triola (1999) apresenta duas importantes vantagens do uso de testes não-paramétricos, relevantes para este estudo: (I) podem ser aplicados a uma ampla diversi- 
dade de situações, porque não dependem das exigências mais rígidas próprias de seus correspondentes paramétricos (como normalidade de distribuição); (2) ao contrário dos testes paramétricos, os testes não-paramétricos podem freqüentemente ser aplicados a dados não-numéricos (como o sexo dos entrevistados). Os testes não-paramétricos utilizados são explicados no decorrer do texto.

As questões elaboradas para medir as atitudes dos estudantes universitários holandeses com relação aos produtos brasileiros se utilizam de cinco categorias, segundo a escala de somatório de Likert, aqui reproduzida. De acordo com Malhotra (1996), quanto maior for o número de categorias, melhor será a discriminação possível entre objetos de estímulo. Porém, como a maioria dos respondentes não consegue lidar com muitas categorias, diretrizes tradicionais sugerem que o número apropriado de categorias em uma escala deve ser sete mais ou menos dois: entre cinco e nove. Optou-se, nesta pesquisa, pela escala de cinco pontos, com o objetivo de simplificar o preenchimento do questionário por parte dos respondentes.

QUADRO 2

ESCALA DE SOMATÓRIO DE LIKERT UTILIZADA NA PESQUISA

\begin{tabular}{ccccc}
\hline 1 & 2 & 3 & 4 & 5 \\
\hline $\begin{array}{c}\text { Discordo } \\
\text { totalmente }\end{array}$ & $\begin{array}{c}\text { Discordo } \\
\text { pouco }\end{array}$ & $\begin{array}{c}\text { Não concordo } \\
\text { nem discordo }\end{array}$ & $\begin{array}{c}\text { Concordo } \\
\text { Pouco }\end{array}$ & $\begin{array}{c}\text { Concordo } \\
\text { totalmente }\end{array}$ \\
\hline
\end{tabular}

Fonte: Elaborado pelos autores.

Finalmente, com relação à coleta dos dados, a opção desta pesquisa de campo foi pela coleta via questionário auto-administrado na forma eletrônica, em razão do grande número de variáveis e do acesso à amostra estudada, pois a populaçãoalvo está localizada em outro país. O questionário foi colocado em uma página de internet para o qual os respondentes, os estudantes de graduação da School of Economics Haarlem, foram direcionados por meio de um link colocado em e-mail convite.

O convite foi reforçado pelos professores dos cursos de graduação, durante as aulas. Essa forma de coleta de dados via internet tem diversas vantagens, como os baixos custos, pois não há despesas com reprodução e envio de questionários, o envio direto das respostas marcadas pelos respondentes para a base de dados, evitando a digitação, e a velocidade com que as respostas são obtidas, usualmente em poucos dias. 


\section{RESULTADOS}

No total, foram recebidas 229 respostas, o que corresponde a uma taxa de resposta de $\mathrm{I} 2,05 \%$. Porém, apenas II 6 foram consideradas válidas para as análises, visto que o restante dos respondentes não preencheu todo o questionário. Dessa forma, é necessário ajustar a margem de erro da pesquisa, uma vez que a amostra obtida foi menor do que a inicialmente idealizada (33I respondentes). Ajustando a margem de erro para o tamanho obtido da amostra e considerando a regra de ajuste para população finita, a amostra de Iı 6 respondentes foi analisada com margem de erro de $9 \%$ e nível de confiança de $95 \%$.

Com relação ao perfil da amostra, quase a totalidade dos respondentes é composta por estudantes com até i8 anos (99,1\%). A maioria (54,3\%) é do sexo masculino e possui algum conhecimento sobre o Brasil. Como pode ser verificado na Tabela I, apenas II, $2 \%$ declararam não saber nada sobre o Brasil.

\section{TABELA I}

CONHECIMENTO DOS RESPONDENTES SOBRE O BRASIL

\begin{tabular}{lcccc}
\hline & FREQÜENCIA & PERCENTUAL & $\begin{array}{c}\text { PERCENTUAL } \\
\text { VÁLIDO }\end{array}$ & $\begin{array}{c}\text { PERCENTUAL } \\
\text { ACUMULADO }\end{array}$ \\
\hline Já estive no Brasil & 17 & 14,7 & 14,7 & 14,7 \\
\hline Já li sobre o Brasil & 42 & 36,2 & 36,2 & 50,9 \\
\hline Ouvi falar sobre o Brasil & 44 & 37,9 & 37,9 & 88,8 \\
\hline Não sei nada sobre o Brasil & 13 & 11,2 & 11,2 & 100,0 \\
\hline Total & 116 & 100,0 & 100,0 & \\
\hline
\end{tabular}

Fonte: Dados coletados na pesquisa.

A identificação de valores extremos e de valores inesperados é um procedimento importante quando do exame da base de dados da pesquisa empírica, pois os resultados das análises podem ser comprometidos se tais valores permanecerem na base de dados.

Com relação aos valores inesperados, os dados foram examinados por meio da observação das tabelas de freqüência e da ferramenta "missing value analysis" do software estatístico SPSS, para identificar valores inesperados ou faltantes. Porém, como o questionário foi colocado em uma página na internet, na qual 
havia um comando que não permitia que respondentes passassem para a próxima página do questionário sem responder a todas as questões fechadas, não houve valores faltantes ou inesperados nessas questões.

Para verificar se as atitudes dos respondentes com relação aos produtos brasileiros são positivas ou negativas, foram efetuados os seguintes procedimentos. Primeiramente, criaram-se variáveis representando a atitude em relação a cada um dos quatro produtos investigados (carne bovina, frutas frescas, calçados e móveis), por meio da soma das respostas dos respondentes às onze questões apresentadas no Quadro I. A Tabela 2 mostra as estatísticas descritivas para as variáveis, representando as atitudes dos respondentes com relação aos produtos brasileiros.

Como a escala possui cinco pontos, e são onze frases avaliadas para cada produto, o valor máximo que poderia ser obtido seria 55 pontos (representando o nível máximo de atitude), o mínimo igual a onze pontos (representando o nível mínimo de atitude) e o valor médio igual a 33 pontos.

TABELA 2

ESTATÍSTICAS DESCRITIVAS DAS ATITUDES COM RELAÇÃO AOS PRODUTOS BRASILEIROS

\begin{tabular}{lcccccc}
\hline & N & MÍNIMO & MÁXIMO & MEDIANA & MÉDIA & DESVIO PADRÃO \\
\hline Atitude carne & 116 & 15,00 & 51,00 & 34 & 33,46 & 5,98 \\
\hline Atitude frutas & 116 & 27,00 & 49,00 & 35 & 36,00 & 4,27 \\
\hline Atitude calçados & 116 & 18,00 & 45,00 & 30 & 30,59 & 3,91 \\
\hline Atitude móveis & 116 & 26,00 & 41,00 & 33 & 33,09 & 2,27 \\
\hline
\end{tabular}

Fonte: Dados coletados na pesquisa.

Observa-se na Tabela 2 que, como os valores da média e da mediana das variáveis não são os mesmos, a distribuição das respostas não tende à normalidade. Para verificar essa hipótese, executa-se um teste Kolmogorov-Smirnov, cujos resultados são mostrados na Tabela 3. De acordo com a Tabela 3, verifica-se que, realmente, as distribuições das respostas às variáveis da pesquisa não possuem distribuição normal. A probabilidade da estatística $Z$ está abaixo de 0,05 , em todos os quatro casos, o que significa que a distribuição normal não é um bom ajuste para os dados na amostra. 


\section{TABELA 3}

TESTE DE NORMALIDADE DE KOLMOGOROV-SMIRNOV

\begin{tabular}{lrrrrr}
\hline & & $\begin{array}{r}\text { ATITUDE } \\
\text { CARNE }\end{array}$ & $\begin{array}{r}\text { ATITUDE } \\
\text { FRUTAS }\end{array}$ & $\begin{array}{r}\text { ATITUDE } \\
\text { CALÇADOS }\end{array}$ & $\begin{array}{r}\text { ATITUDE } \\
\text { MÓVEIS }\end{array}$ \\
\hline N & Média & 33,46 & 36,00 & 30,59 & 33,09 \\
\hline Parâmetros normais & Desvio padrão & 5,98 & 4,27 & 3,91 & 2,27 \\
\hline & Absoluto & 0,185 & 0,170 & 0,157 & 0,163 \\
\hline Diferenças mais extremas & Positivo & 0,130 & 0,170 & 0,157 & 0,163 \\
\hline & Negativo & $-0,185$ & $-0,129$ & $-0,112$ & $-0,156$ \\
\hline Z Kolmogorov-Smirnov & & 1,99 & 1,83 & 1,69 & 1,76 \\
\hline Sig. (bicaudal) & & 0,001 & 0,002 & 0,007 & 0,004 \\
\hline
\end{tabular}

Fonte: Dados coletados na pesquisa.

Considerando que as distribuições das respostas às variáveis da pesquisa não possuem distribuição normal e que a escala de mensuração utilizada não é intervalar, para verificar se existem diferenças estatisticamente significativas entre as atitudes dos respondentes com relação aos produtos brasileiros investigados, será necessário realizar testes não-paramétricos.

O primeiro teste realizado é o teste de Friedman, que é uma espécie de análise de variância a dois critérios de variação, para dados amostrais vinculados. Como os mesmos respondentes avaliaram os quatro produtos brasileiros, as amostras são consideradas não-independentes, sendo necessário usar um teste para amostras relacionadas (SPSS, 1998). A hipótese nula, nesse caso, é de que não existem diferenças nas atitudes com relação aos quatro produtos brasileiros.

A Tabela 4 mostra a média dos postos para as atitudes com relação a cada produto brasileiro analisado. A atitude com relação às frutas é a mais alta, seguida pela carne bovina, móveis e, por último, calçados. O teste de Friedman examina se essas diferenças são estatisticamente significativas (ver Tabela 5). 


\section{TABELA 4}

ORDENAÇÃO DAS MÉDIAS PARA OS PRODUTOS BRASILEIROS

\begin{tabular}{lc}
\hline & ORDENAÇÃO \\
\hline Atitude carne & 2,66 \\
\hline Atitude frutas & 3,28 \\
\hline Atitude calçados & 1,49 \\
\hline Atitude móveis & 2,56 \\
\hline
\end{tabular}

Fonte: Dados coletados na pesquisa.

Conforme mostra a Tabela 5, pode-se rejeitar a hipótese nula de que não existem diferenças entre as atitudes dos respondentes com relação aos produtos brasileiros. Isso significa que pelo menos uma das variáveis tem valores estatisticamente diferentes das outras. Para saber se todas as variáveis são diferentes, ou se apenas uma o é, deve-se realizar o teste Wilcoxon para duas amostras de cada vez, analogamente ao teste t, mas para análises não-paramétricas (SPSS, I998).

\section{TABELA 5}

TESTE DE FRIEDMAN PARA OS PRODUTOS BRASILEIROS

\begin{tabular}{lr}
\hline \multicolumn{1}{c}{$N$} & \multicolumn{1}{c}{116} \\
\hline Qui-quadrado & 123,697 \\
\hline Graus de liberdade & 3 \\
\hline Sig. & 0,000 \\
\hline
\end{tabular}

Fonte: Dados coletados na pesquisa.

Os resultados são mostrados na Tabela 6. Pode-se concluir que todos os pares são estatisticamente diferentes, com exceção da atitude com relação aos móveis e atitude com relação à carne. Isso significa que os respondentes têm, em geral, as mesmas avaliações dos móveis e da carne brasileira.

Sabendo que as atitudes em geral com relação aos produtos são diferentes umas das outras, com exceção dos móveis e da carne bovina, outra análise a ser realizada é verificar quantas pessoas têm uma atitude favorável, neutra ou desfavorável com relação a esses produtos. Para tanto, devem-se definir as categorias: a categoria "atitude desfavorável" compreende os valores da escala somada entre 
II e 32 pontos, a categoria "atitude favorável" compreende os valores entre 34 e 55 pontos, e a categoria neutra é representada pelo valor 33 .

\section{TABELA 6}

TESTE WILCOXON PARA AS VARIÁVEIS DE ATITUDE

COM RELAÇÃO A PRODUTOS BRASILEIROS

\begin{tabular}{lcccccc}
\hline & ATITUDE & ATITUDE & ATITUDE & ATITUDE & ATITUDE & ATITUDE \\
& FRUTAS - & CALÇADOS & MÓVEIS - \\
ATITUDE & CALÇADOS & MÓVEIS - & MÓVEIS - \\
CARNE & CARNE & ATITUDE & CARNE & $\begin{array}{c}\text { ATITUDE } \\
\text { FRUTAS }\end{array}$ & $\begin{array}{c}\text { ATITUDE } \\
\text { FRUTAS }\end{array}$ & $\begin{array}{c}\text { ATITUDE } \\
\text { CALÇADOS }\end{array}$ \\
\hline Z & $-4,067$ & $-5,471$ & $-0,826$ & $-8,835$ & $-6,264$ & $-6,422$ \\
\hline Sig. (bicaudal) & 0,000 & 0,000 & 0,409 & 0,000 & 0,000 & 0,000 \\
\hline
\end{tabular}

Fonte: Dados coletados na pesquisa.

A partir das tabelas de freqüências de cada variável, foram elaborados os Gráficos de I a 4, mostrando a porcentagem dos respondentes que pertencem a cada categoria já mencionada. Com relação à carne bovina, 54,31\% dos respondentes têm atitudes favoráveis, I7,24\% atitudes neutras e $28,45 \%$ atitudes negativas.

Comparando todos os produtos, verifica-se que as frutas frescas têm a maior porcentagem de atitudes favoráveis $(72,40 \%)$ e os calçados apresentam a avaliação mais baixa (apenas I8,97\% dos respondentes têm atitudes favoráveis a esse produto e $76,72 \%$ atitudes desfavoráveis). O produto com mais avaliações neutras são os móveis, com 31,90\% das respostas na categoria neutra (valor 33).

\section{GRÁFICO I}

\section{ATITUDE COM RELAÇÃO A CARNE BOVINA BRASILEIRA}

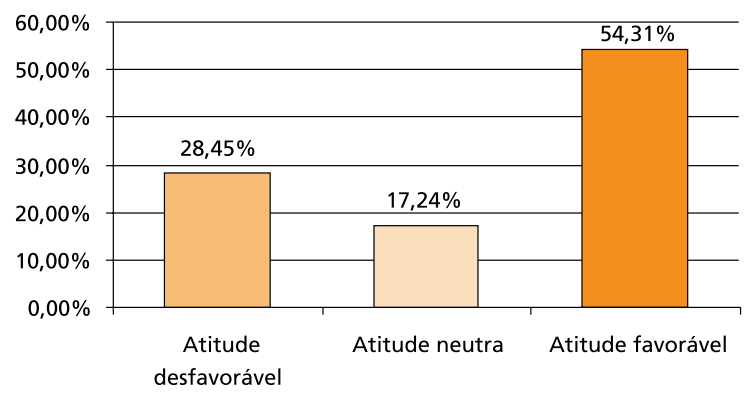

Fonte: Dados coletados na pesquisa. 


\section{GRÁFICO 2}

ATITUDE COM RELAÇÃO AS FRUTAS FRESCAS BRASILEIRAS

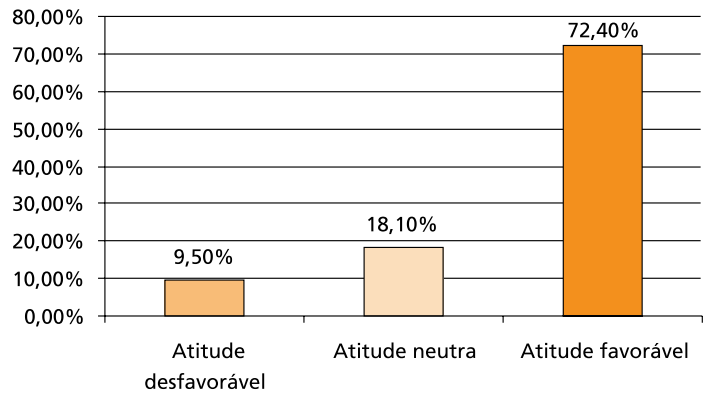

Fonte: Dados coletados na pesquisa.

\section{GRÁFICO 3}

\section{ATITUDE COM RELAÇÃO AOS CALÇADOS BRASILEIROS}

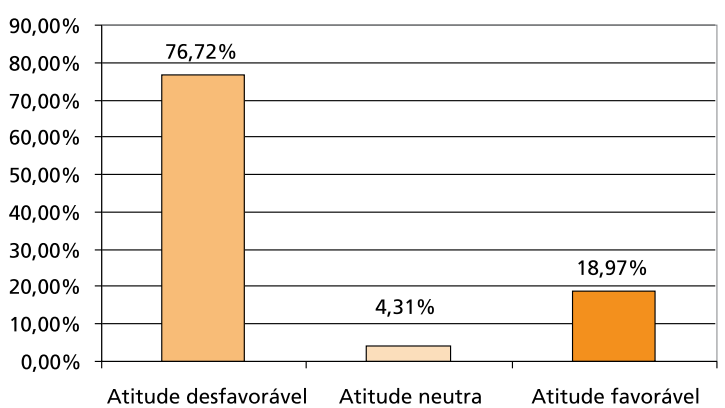

Fonte: Dados coletados na pesquisa.

\section{GRÁFICO 4}

ATITUDE COM RELAÇÃO AOS MÓVEIS BRASILEIROS

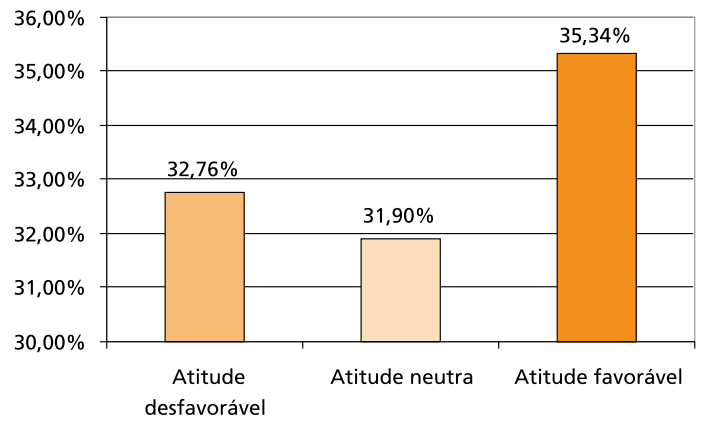

Fonte: Dados coletados na pesquisa. 
Nesse momento, é interessante verificar se existem diferenças nas atitudes com relação aos produtos analisados, dependendo do grau de conhecimento dos respondentes sobre esses produtos. Dessa forma, pode ser verificado se o grau de conhecimento influencia as avaliações que os respondentes têm dos produtos brasileiros. O questionário utilizado perguntava o quanto os respondentes conheciam de carne bovina, frutas frescas, calçados e móveis brasileiros em uma escala de I ("conheço muito bem") a 5 ("não conheço nada").

Assim, é efetuado um teste de Kruskal-Wallis, uma versão não-paramétrica da análise de variância para amostras independentes, que usa os postos dos valores originais e não os valores em si (SPSS, I998). A estatística do teste de Kruskal-Wallis mede o quanto os postos do grupo diferem da ordenação média de todos os grupos. As tabelas seguintes mostram os resultados obtidos. Na Tabela 7, têm-se os postos para as categorias das variáveis "se conhece carne brasileira" e "atitude carne".

\section{TABELA 7}

POSTOS PARA CARNE BOVINA BRASILEIRA

\begin{tabular}{cccc}
\hline & SE CONHECE CARNE BRASILEIRA & N & ORDENAÇÃO \\
\hline Categorias de atitude carne & 1 & 13 & 48,38 \\
\hline 2 & 18 & 65,28 \\
\hline 3 & 29 & 60,52 \\
\hline 5 & 13 & 67,00 \\
\hline Total & 43 & 54,79 \\
\hline
\end{tabular}

Fonte: Dados coletados na pesquisa.

A Tabela 8 mostra os resultados do teste. É estimada a probabilidade de obter uma estatística qui-quadrado maior ou igual a 4,I46, se realmente não existirem diferenças entre os postos do grupo. Nesse caso, esse valor de quiquadrado, com 4 graus de liberdade, deveria ocorrer 387 vezes em I.000. Por isso, conclui-se que não existem diferenças estatisticamente significativas na atitude com relação à carne bovina, dependendo do conhecimento dos respondentes sobre esse produto. 


\section{TABELA 8}

TESTE DE KRUSKAL-WALLIS PARA CARNE BOVINA BRASILEIRA

\begin{tabular}{lc}
\hline & $\begin{array}{c}\text { CATEGORIAS DE } \\
\text { ATITUDE CARNE }\end{array}$ \\
\hline Qui-quadrado & 4,146 \\
\hline Graus de liberdade & 4 \\
\hline Sig. & 0,387 \\
\hline
\end{tabular}

Fonte: Dados coletados na pesquisa.

Ao analisar as Tabelas de 9 a I4, verifica-se que apenas para os calçados brasileiros existiu uma diferença estatisticamente significativa para a atitude com relação ao produto entre os níveis de conhecimento desse produto. Para frutas frescas e móveis brasileiros, não houve diferença na atitude entre os grupos de respondentes com mais ou menos conhecimento dos produtos.

\section{TABELA 9}

POSTOS PARA FRUTAS FRESCAS BRASILEIRAS

\begin{tabular}{lccc}
\hline & $\begin{array}{l}\text { SE CONHECE FRUTAS } \\
\text { FRESCAS BRASILEIRAS }\end{array}$ & ORDENAÇÃO \\
\hline Categorias de atitude frutas & 1 & 17 & 56,24 \\
\hline & 2 & 29 & 57,81 \\
\hline 3 & 35,59 & 71,00 \\
\hline 4 & 35 & 15 & 51,90 \\
\hline
\end{tabular}

Fonte: Dados coletados na pesquisa. 
- RAM - REVISTA DE ADMINISTRAÇÃO MACKENZIE •

Volume 9, n. 3, 2008, p. 11-37

\section{TABELA IO}

TESTE DE KRUSKAL-WALLIS PARA FRUTAS FRESCAS BRASILEIRAS

\begin{tabular}{lc}
\hline & $\begin{array}{c}\text { CATEGORIAS DE } \\
\text { ATITUDE FRUTAS }\end{array}$ \\
\hline Qui-quadrado & 4,779 \\
\hline Graus de liberdade & 4 \\
\hline Sig. & 0,311 \\
\hline
\end{tabular}

Fonte: Dados coletados na pesquisa.

\section{TABELA II}

POSTOS PARA CALÇADOS BRASILEIROS

\begin{tabular}{cccc}
\hline $\begin{array}{c}\text { SE CONHECE } \\
\text { CALÇADOS BRASILEIROS }\end{array}$ & N & ORDENAÇÃO \\
\hline Categorias de atitude calçados & 1 & 14 & 66,61 \\
\hline 2 & 15 & 72,33 \\
\hline 3 & 20 & 56,42 \\
\hline 4 & 25 & 65,70 \\
\hline & 5 & 42 & 47,56 \\
\hline
\end{tabular}

Fonte: Dados coletados na pesquisa.

\section{TABELA I2}

TESTE DE KRUSKAL-WALLIS PARA CALÇADOS BRASILEIROS

\begin{tabular}{lc}
\hline & $\begin{array}{c}\text { CATEGORIAS DE } \\
\text { ATITUDE CALÇADOS }\end{array}$ \\
\hline Qui-quadrado & 16,655 \\
\hline Graus de liberdade & 4 \\
\hline Sig. & 0,002 \\
\hline
\end{tabular}

Fonte: Dados coletados na pesquisa. 


\section{TABELA I3}

POSTOS PARA MÓVEIS BRASILEIROS

\begin{tabular}{ccccc}
\hline & $\begin{array}{c}\text { SE CONHECE } \\
\text { MÓVEIS BRASILEIROS }\end{array}$ & N & ORDENAÇÃO \\
\hline Categorias de atitude móveis & 1 & 6 & 44,75 \\
\hline 2 & 13 & 63,46 \\
\hline 4 & 28 & 53,52 \\
\hline 5 & 63 & 59,88 \\
\hline
\end{tabular}

Fonte: Dados coletados na pesquisa.

\section{TABELA I4}

TESTE KRUSKAL-WALLIS PARA MÓVEIS BRASILEIROS

\begin{tabular}{lc}
\hline & $\begin{array}{c}\text { CATEGORIAS DE } \\
\text { ATITUDE MÓVEIS }\end{array}$ \\
\hline Qui-quadrado & 2,925 \\
\hline Graus de liberdade & 4 \\
\hline Sig. & 0,570 \\
\hline
\end{tabular}

Fonte: Dados coletados na pesquisa.

Porém, assim como a Anova, o teste de Kruskal-Wallis não revela como os grupos de respondentes com mais ou menos conhecimento sobre os calçados brasileiros diferem entre si, mas apenas informa que são diferentes. Por isso, é efetuado o teste de Mann-Whitney para realizar as comparações pareadas entre as amostras independentes (SPSS, I998). Porém, é preciso primeiro agrupar os respondentes em dois grupos, de acordo com o conhecimento que possuem dos calçados brasileiros.

Como a escala usada para medir o quanto os respondentes conheciam os calçados brasileiros variava de i a 5 ("I" sendo "conheço muito bem" e " 5 " sendo "não conheço nada"), foi arbitrado que os valores de I a 3 seriam agrupados na 
categoria "conhece calçados brasileiros" e os valores 4 e 5 seriam agrupados na categoria "não conhece calçados brasileiros". A Tabela I5 mostra os postos para as categorias das variáveis apresentadas.

De acordo com a Tabela i6, os dois grupos de respondentes (que conhecem e que não conhecem os calçados brasileiros) são diferentes entre si, ao nível de confiança de $95 \%$. Os respondentes que mais conhecem os calçados brasileiros possuem uma atitude mais favorável a esses produtos do que aqueles que os conhecem menos.

\section{TABELA I5}

POSTOS PARA CONHECIMENTO DE CALÇADOS BRASILEIROS

\begin{tabular}{rcccc}
\hline & $\begin{array}{c}\text { CATEGORIAS DE } \\
\text { CONHECIMENTO } \\
\text { DE CALÇADOS }\end{array}$ & ORDENAÇÃO & SOMA \\
\hline $\begin{array}{r}\text { Categorias de } \\
\text { atitude calçados }\end{array}$ & Conhece calçado & 49 & 64,20 & 3146,00 \\
\hline & Não conhece calçado & 67 & 54,33 & 3640,00 \\
\hline Total & 116 & & \\
\hline
\end{tabular}

Fonte: Dados coletados na pesquisa.

\section{TABELA I6}

TESTE DE MANN-WHITNEY PARA CALÇADOS BRASILEIROS

\begin{tabular}{lc}
\hline & $\begin{array}{c}\text { CATEGORIAS DE } \\
\text { ATITUDE CALÇADOS }\end{array}$ \\
\hline Mann-Whitney U & 1362,000 \\
\hline Wilcoxon W & 3640,000 \\
\hline Z & $-2,123$ \\
\hline Sig. (bicaudal) & 0,034 \\
\hline
\end{tabular}

Fonte: Dados coletados na pesquisa.

A seguir, apresentam-se as considerações finais do artigo, mostrando as conclusões e contribuições do trabalho, limitações da pesquisa e algumas possibilidades para futuros estudos na área. 


\section{CONCLUSÕES}

O presente trabalho buscou verificar se existem diferenças nas atitudes que um segmento de consumidores estrangeiros possui em relação aos seguintes produtos brasileiros: carne bovina, frutas frescas, calçados e móveis. A pesquisa de campo analisou a população de estudantes holandeses da School of Economics Haarlem (Holanda).

Os resultados apontam que os produtos brasileiros analisados possuem diferentes avaliações dos respondentes, com exceção da atitude com relação aos móveis e atitude com relação à carne bovina, que apresentam atitudes estatisticamente iguais entre si. Os produtos que possuem as melhores avaliações, ou seja, atitudes mais favoráveis, são as frutas frescas. Por sua vez, os calçados são os produtos que receberam as avaliações mais negativas por parte dos consumidores entrevistados.

O estudo, no entanto, também mostrou que os calçados recebem avaliações mais positivas daqueles respondentes que disseram conhecer o produto. Portanto, percebe-se que, como a maioria dos entrevistados não conhece os calçados brasileiros, no geral a avaliação do produto é desfavorável. Para os demais produtos brasileiros, não foram encontradas diferenças estatisticamente significativas entre os respondentes que conhecem mais ou menos esses produtos. Tal achado sugere que os calçados brasileiros, talvez por se tratar de uma categoria de produto cujo consumo tem grande exposição social, podem se beneficiar de esforços de comunicação mais que os outros produtos pesquisados.

Com relação às limitações da pesquisa, pode ser destacada a população-alvo definida para este estudo. A população da pesquisa não abrange outros importantes mercados com os quais o Brasil mantém relações comerciais, como outros países europeus, os Estados Unidos e a China, ou mesmo outros segmentos de consumidores holandeses. A população foi definida de uma forma restrita, o que impede a generalização dos resultados obtidos para outros segmentos de consumidores. Os resultados obtidos nesta pesquisa refletem apenas a opinião dessa população de estudantes específica.

Outros aspectos referem-se ao método da pesquisa de campo. A relativa falta de conhecimento sobre o Brasil pode ter se refletido no baixo aproveitamento das respostas obtido na pesquisa. Dos I.9or estudantes de graduação da School of Economics Haarlem, que receberam um em e-mail convite para a pesquisa, 229 responderam, o que corresponde a uma taxa de resposta de ı2,05\%. Porém, apenas II6 questionários foram considerados válidos para as análises, visto que o restante dos respondentes não preencheu todo o questionário.

Em vista desses fatos, estudos futuros poderiam verificar as atitudes que outros segmentos de consumidores possuem sobre os produtos brasileiros, além 
de incluir outros produtos nos estudos. Além disso, outras pesquisas poderiam buscar levantar hipóteses sobre os antecedentes da atitude com relação aos produtos brasileiros, como o grau de conhecimento e familiaridade com os produtos e com o país.

De forma geral, verifica-se que o conhecimento das atitudes que consumidores estrangeiros têm dos produtos brasileiros pode levar à identificação de pontos fracos e fortes na comercialização desses produtos no exterior. Ao entender melhor o comportamento do consumidor (nesse caso, suas atitudes), os profissionais de marketing podem oferecer produtos que satisfaçam diretamente as suas necessidades, podem usar as atitudes para segmentar mercados e também desenvolver estratégias promocionais mais efetivas.

\section{REFERÊNCIAS}

AAKER, D. A.; DAY, G. S. Marketing research. 2. ed. New York: John Wiley, I983.

AGBONIFOH, B. A.; ELIMIMIAN, J. U. Attitudes of developing countries towards "country-oforigin" products in an era of multiple brands. Journal of International Consumer Marketing, New York, v. II, n. 4, p. 97-II6, I999.

ALICEWEB - Sistema de Análise das Informações de Comércio Exterior via Internet. Secretaria de Comércio Exterior (SECEX). Ministério do Desenvolvimento, Indústria e Comércio Exterior. Disponível em: <http://aliceweb.desenvolvimento.gov.br/>. Acesso em: ı.․o abr. 2005 .

ASSAEL, H. Consumer behavior and marketing action. Cincinnati: South-Western College Publishing, I995.

AYROSA, E. A. T. Some notes on the development of research on country-of-origin effects. Arché Internacional, Rio de Janeiro, ano IX, v. 26, 2000.

Validation of a scale to measure country image in Brazil. In: BALAS - THE BUSINESS ASSOCIATION OF LATIN AMERICAN STUDIES ANNUAL CONFERENCE, 2002, São Paulo. Anais... São Paulo, 2002. CD-ROM.

BALABANIS, G.; MUELLER, R.; MELEWAR, T. C. Country of Origin Images around the World: Can Value Priorities Predict Them? In: 28th EMAC CONFERENCE, I999, Berlin. Proceedings... Berlin, I999. CD-ROM.

BLACKWELL, R. D.; MINIARD, P. W.; ENGEL, J. F. Comportamento do consumidor. São Paulo: Pioneira Thomson Learning, 2005.

BLUM, M. L.; NAYLOR, J. C. Psicología industrial: sus fundamentos teóricos y sociales. México: Trillas, I976.

CHAO, P.; GUPTA, P. B. Information search and efficiency of consumer choices of new cars: country-of-origin effects. International Marketing Review, London, v. I2, n. 6, p. 47-59, I995. 
CHURCHILL, G. A. Marketing research: methodological foundations. Fort Worth: The Dryden Press, I99I.

CORDELL, V. V. Effect of consumer preferences for foreign sourced products. Journal of International Business Studies, London, v. 23, n. 2, p. 25-30, 1993.

DAY, G. S. Evaluating models of attitude structure. Journal of Marketing Research, v. 9, p. 279-286, I972.

FISHBEIN, M.; AJZEN, I. Belief, attitude, intention and behavior: an introduction to theory and research. Reading: Addison-Wesley, I975.

HAIR, J. F. et al. Multivariate data analysis. Upper Saddle River: Prentice Hall, I995.

KARSAKLIAN, E. Comportamento do consumidor. São Paulo: Atlas, 2004.

MALHOTRA, N. K. Marketing research: an applied orientation. Upper Saddle River: Prentice Hall, I996.

MATTAR, F. N. Pesquisa de marketing: metodologia e planejamento. São Paulo: Atlas, I999.

MCDANIEL, C.; GATES, R. Contemporary marketing research. Saint Paul: West Publishing Company, I996.

NEBENZAHL, I. D.; JAFFE, E. D.; SHLOMO, I. L. Towards a theory of country image effect on product evaluation. Management International Review, Wiesbaden, v. 37, n. I, p. 27-49, I997.

NETEMEYER, R.; DURVASULA, S.; LICHTENSTEIN, D. A cross-national assessment of the reliability and validity of the CETSCALE. Journal of Marketing Research, v. 28, p. 320-327, August I99I.

RECEITA FEDERAL. Ministério da Fazenda do Brasil. Disponível em: <http://www.receita. fazenda.gov.br>. Acesso em: I8 maio 2004.

RODRIGUES, A. Psicologia social. 6. ed. Petrópolis: Vozes, I977.

SCHIFFMAN, L. G.; KANUK, L. L. Consumer behavior. Upper Saddle River: Prentice Hall, I997. $672 \mathrm{p}$.

SIEGEL, S. Estatística não-paramétrica para as ciências do comportamento humano. São Paulo: McGraw-Hill, I975.

SPSS Base 8. Applications guide. Chicago: SPSS Inc., I998.

TAGLIACARNE, G. Pesquisa de mercado: técnica e prática. São Paulo: Atlas, I989.

TRIOLA, M. F. Introdução à estatística. Rio de Janeiro: LTC, I999.

WILKIE, W. L. Consumer behavior. New York: John Wiley, I994.

\section{TRAMITAÇ ÃO}

Recebido em 2/8/2006

Aprovado em 6/3/2008 